Chronic Obstructive Pulmonary Diseases:

Journal of the COPD Foundation

Original Research

\title{
Risk of Death by Comorbidity Prompting Rehospitalization Following the Initial COPD Hospitalization
}

\author{
Sean Duffy, MD, Shari Barnett, MD, Brian Civic, MD, A. James Mamary, MD, Gerard J. Criner, MD ${ }^{1}$
}

\begin{abstract}
Rationale: Chronic obstructive pulmonary disease (COPD) hospitalizations increase short and long-term mortality; multiple COPD hospitalizations track with even higher mortality. While comorbidities such as coronary artery disease (CAD) and congestive heart failure (CHF) are common in COPD, their contribution to mortality risk after a sentinel COPD hospitalization is unknown.

Purpose: Assess the effect on mortality of comorbid conditions prompting rehospitalization following COPD exacerbation hospitalization.

Methods: We performed a retrospective cohort analysis of patients hospitalized for COPD exacerbations in Pennsylvania from 1990-2010 using the Pennsylvania Health Care Cost Containment Council (PHC4) database. We included patients $>40$ years old hospitalized for an acute exacerbation of COPD (AECOPD; International Classification of Diseases-Ninth Edition, [ICD-9] \#491, 492, 496) by discharge diagnosis. Thirty-day mortality in patients with COPD hospitalization for acute exacerbation who were rehospitalized for COPD < 30days post-discharge was compared to those primarily readmitted for comorbid conditions. Relative risk of death after readmission was determined by diagnosis. Primary end-point was mortality 30 days post-readmission for 14 most common nonCOPD diagnoses, including heart failure, pneumonia, pulmonary embolus (PE), and myocardial infarction.

Results: Patients were nearly 2 times more likely to die within 30 days when readmitted for pneumonia $(p<0.0001)$ or myocardial infarction $(\mathrm{p}<0.0001)$ rather than COPD. Septicemia conferred the highest mortality.

Conclusions: COPD patients rehospitalized for comorbid conditions such as myocardial infarction, pneumonia, septicemia or pulmonary heart disease (includes PE) were significantly more likely to die within 30 days than patients readmitted for COPD. Great emphasis is already placed on preventing COPD rehospitalization; however, more attention should focus on preemptive risk reduction for comorbidities in COPD patients.
\end{abstract}

Abbreviations: coronary artery disease, CAD; congestive heart failure, CHF; pulmonary embolism, PE; Pennsylvania Health Care Cost Containment Council, PHC4; acute exacerbation of COPD, AECOPD; COPD specific cOmorbidity TEst, COTE; International Classification of Diseases-Ninth Edition, ICD-9; odds ratio, OR; community-acquired pneumonia, CAP

Funding Support: Funding provided by the Maxine Gitlin Lung Research Fund.

Date of Acceptance: August 14, 2014

Citation: Duffy S, Barnett S, Civic B, Mamary AJ, Criner GJ. Risk of death by comorbidity prompting rehospitalization following the initial COPD hospitalization. J COPD F. 2015;2(1): 17-22. http://dx.doi.org/10.15326/jcopdf.2.1.2014.0129.

1 Division of Pulmonary and Critical Care Medicine, Temple University School of Medicine, Philadelphia, Pennsylvania

Keywords:

COPD; hospitalization; comorbidity

\section{Address correspondence to:}

Sean Duffy, M.D.

Division of Pulmonary and Critical Care Medicine

Temple University School of Medicine

745 Parkinson Pavilion

3401 North Broad Street

Philadelphia, PA, 19140

Phone: (215)707-5864

Fax (215)707-6867

Email: sean.duffy2@tuhs.temple.edu 


\section{Introduction}

The diagnosis of chronic obstructive pulmonary disease (COPD) carries with it a large health burden including medication prescription, outpatient and inpatient costs. In 1998, COPD was responsible for an estimated 14.2 million ambulatory visits and 1.4 million emergency department visits. ${ }^{1}$ One study examining Medicare patients with and without COPD reported that COPD patients were 2.3 times more likely to be hospitalized once during the year, had longer hospital admissions, and incurred nearly twice the annual health care costs. ${ }^{2}$

COPD patients carry significantly more comorbid diagnoses than the general public; a factor that likely contributes to their increased mortality. Comorbid conditions include coronary artery disease (CAD), congestive heart failure (CHF), pneumonia, pulmonary embolus (PE), muscle wasting, depression, reduced fat-free mass, osteopenia, and chronic infections lead to an early mortality in patients with COPD. ${ }^{3}$ In fact, prior studies have shown that the majority of COPD patients die from comorbid conditions and subsequent complications rather than COPD. ${ }^{4}$

Besides being more prevalent in COPD, specific comorbidities are associated with a higher mortality. For instance, cardiovascular disorders and diabetes were found to have a greater impact on mortality than essential hypertension. ${ }^{5}$ Several multidisciplinary scores that include pulmonary and non-pulmonary factors have been used to predict mortality in COPD. Of note, the COPD specific cOmorbidity TEst (COTE) focuses on the contribution of comorbid diagnoses to mortality in COPD patients. ${ }^{6}$ The COTE index found that $\mathrm{CAD}, \mathrm{CHF}$, anxiety, pulmonary hypertension, cancer (including lung, breast, pancreatic and esophageal), gastric/duodenal ulcers, diabetes with neuropathy, liver cirrhosis, gout, and ventricular arrhythmia all carry increased mortality but did not examine the impact of hospitalizations for these comorbidities on short term mortality. ${ }^{7}$

Thirty-day readmission is a new area of focus since many patients rehospitalized soon after hospital discharge may have remediable factors that if appropriately addressed could improve patient outcome. This paper aims to examine the mortality impact of 30-day rehospitalization for comorbid conditions following an initial COPD exacerbation that required hospitalization. In concordance with previous evaluations, we hypothesize that short-term mortality will be increased in patients rehospitalized with comorbid conditions within 30-days post hospital discharge.

\section{Methods}

We performed a retrospective cohort analysis of all patients hospitalized for COPD exacerbations in Pennsylvania from 1990-2010 using the Pennsylvania Health Care Cost Containment Council database (PHC4). The PHC4 database was used to identify patients with hospitalizations between the years of 19902010 who were hospitalized and carried a discharge diagnosis of COPD. We included Pennsylvania residents at least 40 years old hospitalized for acute exacerbation of COPD. The diagnosis included the primary diagnoses of the International Classification of Diseases, Ninth Edition (ICD-9) \#491, 492 or 496. We examined if patients were rehospitalized in the following 30 days and noted the primary diagnosis for readmission by ICD-9 code. Primary end-point was mortality 30 days post-readmission for the 14 most common non-COPD diagnoses. Comorbid diagnoses included: heart failure, pneumonia, acute renal failure, pulmonary embolus, asthma, atrial fibrillation and myocardial infarction. We employed chi squared and 2-tailed t-tests to analyze the data. Odds ratios (OR) were used to compare 30day mortality of primary comorbid admission to that of patients rehospitalized within 30 days for a subsequent COPD exacerbation prompting rehospitalization.

\section{Results}

The total number of COPD hospitalizations between 1990 and 2010 was 581,590 . Almost $25 \%$ of patients $(140,454)$ required rehospitalization within 30 days of an index COPD hospitalization. About one-third $(48,281)$ of these rehospitalizations were for COPD while two-thirds $(92,173)$ were rehospitalized for a comorbid diagnosis. Of those, the 14 most prevalent comorbid rehospitalization diagnoses were analyzed in this study. Total rehospitalizations and patient demographics are listed by comorbid diagnosis in Table 1.

Patients hospitalized for COPD exacerbation without a 30-day rehospitalization for any cause had a 30-day mortality rate of 7.3 percent. The 30 -day mortality was substantially increased if the patient was rehospitalized within 30 days and varied significantly by the primary diagnosis for rehospitalization. The 30-day mortality for 


\section{Table 1. Demographic Characteristics and Total Number of 30-day Rehospitalizations by Comorbid Diagnosis.}

\begin{tabular}{|c|c|c|c|c|c|c|}
\hline $\begin{array}{l}30 \text { Day Readmission } \\
\text { Diagnosis }\end{array}$ & $\mathrm{N}$ & $\begin{array}{c}\text { 30-Day } \\
\text { Mortality } \\
\text { Rate }\end{array}$ & $\begin{array}{l}\text { Mean } \\
\text { Age }\end{array}$ & $\begin{array}{c}\% \\
\text { Male }\end{array}$ & $\begin{array}{c}\% \\
\text { White }\end{array}$ & $\begin{array}{c}\% \\
\text { Black }\end{array}$ \\
\hline $\begin{array}{l}\text { COPD Admits w/c } 30 \\
\text { day Readmission }\end{array}$ & 301,913 & $7.3 \%$ & 71.1 & $44.9 \%$ & $76.7 \%$ & $8.8 \%$ \\
\hline COPD & 48,281 & $12.4 \%$ & 69.7 & $48.9 \%$ & $73.5 \%$ & $11.3 \%$ \\
\hline Pneumonia & 11,522 & $19.6 \%$ & 72.6 & $54.5 \%$ & $77.8 \%$ & $6.2 \%$ \\
\hline Congestive Heart Failure & 8,134 & $14.6 \%$ & 73.6 & $49.2 \%$ & $73.2 \%$ & $10.9 \%$ \\
\hline Acute Respiratory Failure & 6,037 & $34.7 \%$ & 69.6 & $46.1 \%$ & $81.6 \%$ & $12.2 \%$ \\
\hline Asthma & 4,204 & $4.5 \%$ & 64.4 & $36.7 \%$ & $57.6 \%$ & $24.2 \%$ \\
\hline Coronary Artery Disease & 2,258 & $5.0 \%$ & 70.2 & $56.3 \%$ & $83.1 \%$ & $5.9 \%$ \\
\hline Myocardial Infarction & 1,805 & $20.3 \%$ & 73.3 & $52.0 \%$ & $79.2 \%$ & $6.8 \%$ \\
\hline Atrial Fibrillation & 1,432 & $12.6 \%$ & 74 & $46.0 \%$ & $89.3 \%$ & $5.9 \%$ \\
\hline Aspiration Pneumonia & 1,431 & $37.9 \%$ & 77.6 & $55.9 \%$ & $82.4 \%$ & $7.3 \%$ \\
\hline Volume Depletion & 990 & $14.4 \%$ & 75.7 & $42.8 \%$ & $87.2 \%$ & $7.4 \%$ \\
\hline Septicemia & 941 & $46.3 \%$ & 74.9 & $48.1 \%$ & $87.2 \%$ & $7.5 \%$ \\
\hline C. difficile & 919 & $18.7 \%$ & 75.2 & $40.3 \%$ & $92.1 \%$ & $4.5 \%$ \\
\hline Diabetes Mellitus & 858 & $7.7 \%$ & 70.6 & $44.8 \%$ & $74.0 \%$ & $15.7 \%$ \\
\hline Acute Renal Failure & 757 & $24.2 \%$ & 74.8 & $54.3 \%$ & $83.8 \%$ & $11.9 \%$ \\
\hline $\begin{array}{l}\text { Pulmonary Heart Disease } \\
\text { (includes PE) }\end{array}$ & 713 & $18.5 \%$ & 71.9 & $45.9 \%$ & $78.9 \%$ & $9.5 \%$ \\
\hline
\end{tabular}


patients rehospitalized for COPD within 30 days increased to $12.4 \%(\mathrm{OR}=1.79)$. Thirtyday mortality rates for each of the comorbid diagnoses are listed in Table 1 . Odds ratios were employed to compare the 30-day mortality rates of patients rehospitalized for primary comorbid diagnosis to those rehospitalized for COPD. The most common non-COPD rehospitalization diagnosis was pneumonia ( $\mathrm{n}=11,522)$ followed by CHF $(\mathrm{n}=8134)$ and acute respiratory failure $(\mathrm{n}=$ 6037). The remaining diagnoses and number of rehospitalizations are listed in Table 1.

Patients rehospitalized for comorbid conditions such as septicemia, acute respiratory failure, pulmonary heart disease (includes PE), myocardial infarction, aspiration pneumonia or acute renal failure were significantly more likely to die < 30 days from rehospitalization than patients readmitted for COPD exacerbations. Septicemia conferred the greatest risk of death within 30 days of rehospitalization (OR $=6.11)$, followed by aspiration pneumonia $(\mathrm{OR}=4.3)$, acute respiratory failure $(\mathrm{OR}=$ 3.76), acute renal failure $(\mathrm{OR}=2.26)$ and myocardial infarction $(\mathrm{OR}=1.8)$. The 30-day mortality rates for all comorbid diagnoses are shown in Figure 1. Odds ratios are shown in Figure 2.

\section{Discussion}

Our study shows that rehospitalizations for comorbid diseases such as myocardial infarction, heart failure, pneumonia, aspiration pneumonia and PE confer the greater risk for short-term mortality when compared to recurrent hospitalization for COPD exacerbation. Though other studies have shown increased mortality in COPD patients with comorbidities, our study examines specific comorbid readmission diagnosis and short-term mortality after rehospitalization. Additionally, our study analyzes the prevalence of comorbid diagnoses as the primary rehospitalization diagnosis after initial hospitalization for COPD.

Intuitively, several of the diagnoses show increased mortality if the comorbid condition affects the lungs

\section{Figure 1. Thirty-day Mortality Rate by Comorbidity as Primary Diagnosis for Readmission}

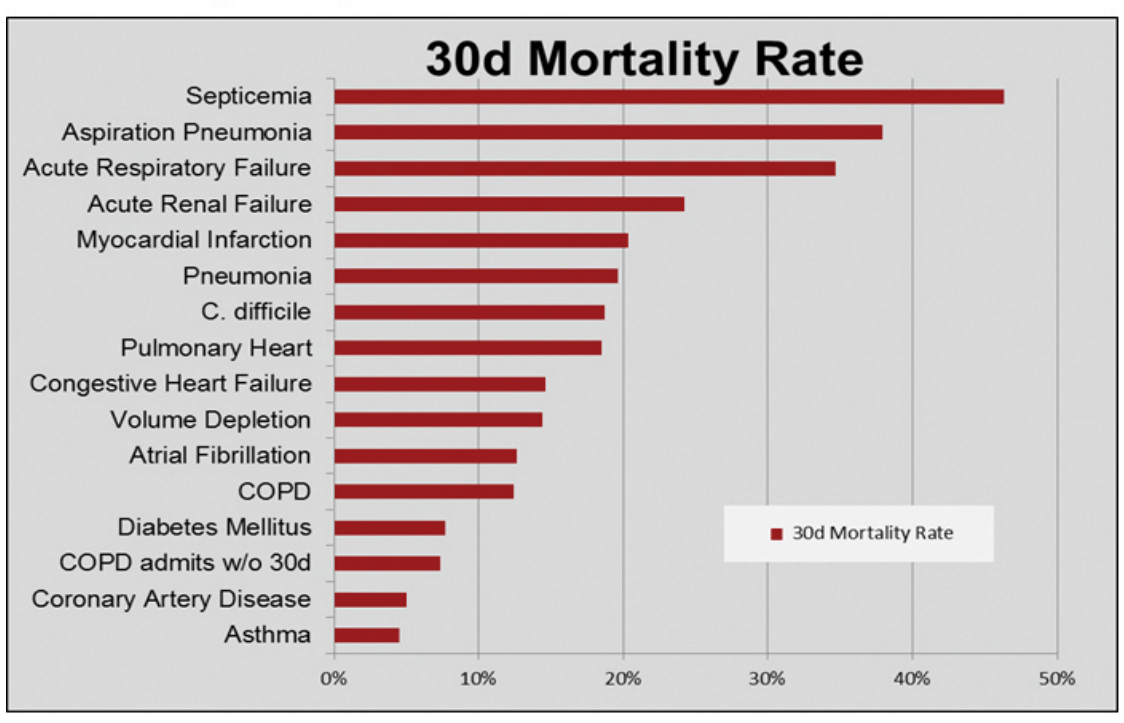

Figure 2. Risk of Death

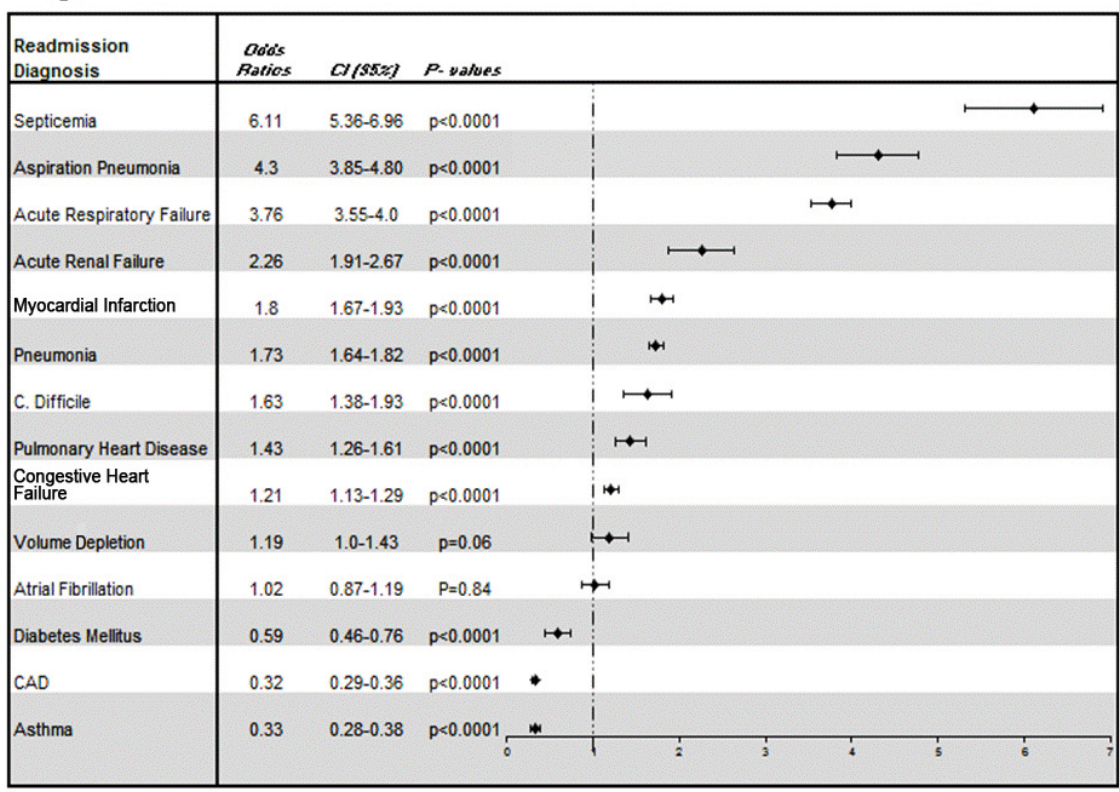

Risk of death at 30 days post discharge for patients readmitted with a primary comorbid diagnosis within 30 days of initial COPD hospitalization as Risk of death at 30 days post discharge for
compared to patients readmitted for COPD. such as acute respiratory failure, aspiration pneumonia, pneumonia, and $\mathrm{PE}$ in patients with underlying COPD. This is likely a result of COPD patients' relatively low respiratory reserve. The consideration of acute respiratory failure as a comorbid condition can be debated as it may be a result of the underlying COPD. Using primary diagnosis codes as identifiers, it is difficult to distinguish patients with acute respiratory failure resulting from COPD from patients suffering from another condition that prompts the development 
of acute respiratory failure.

Other groups have found increased mortality in COPD patients hospitalized with respiratory disorders other than COPD as compared to those without COPD. One study examined the 30-day mortality for patients hospitalized for community-acquired pneumonia (CAP) in patients with and without COPD. ${ }^{8}$ The overall mortality for COPD patients hospitalized with pneumonia was almost $3 \mathrm{x}$ greater than patients who developed CAP but did not have COPD. Additionally these patients were also more likely to develop acute respiratory failure. ${ }^{8}$ Restrepo et al showed increased 30-day and 90-day mortality in COPD patients admitted with CAP as compared to patients without COPD. ${ }^{9}$ Our data supports that those COPD patients rehospitalized with pneumonia are at increased risk of death relative to patients rehospitalized due to recurrent COPD exacerbation.

Our data demonstrates a tendency to identify comorbidities that are associated with an older population. Not surprisingly, aspiration pneumonia occurred in an older population with a mean age $>77$ years. While there was some gender and race predilection to be also seen with other comorbidities it remains unclear if this confers any particular clinical significance.

Given our analysis, we argue that the greatest potential for mortality reduction lies in identifying the rehospitalization diagnoses that may represent a missed or inadequately treated disease process during the index admission rather than a novel disease process. For instance, the development of a myocardial infarction after COPD exacerbation may relate to the physiologic and metabolic stressors that occur during COPD exacerbation. ${ }^{5}$ Given the similarity of symptoms and the ease with which chest pain can be attributed to COPD, clinicians should hold a high index of suspicion for myocardial infarction in these patients. In addition, treatment rendered during the index admission may contribute to some of the rehospitalization diagnoses. For example, extended use of antibiotics may result in C. difficile infection or overuse of diuretics may lead to acute renal failure.

Given the data shown, it is clear that a great emphasis should be placed on controlling exacerbations of COPD as well as managing other comorbid diagnoses for hospitalized COPD patients. Also, thorough evaluations should be performed to prevent readmission for the most deadly and potentially preventable comorbidities like septicemia, aspiration pneumonia, myocardial infarction and acute renal failure.

\section{Study Limitations}

This study has several limitations relating to the PHC4 database. For instance, the diagnoses were obtained based on billing codes for primary diagnosis. These can be inaccurate at times. In addition, the database does not allow for a more thorough review of patient-specific characteristics at the time of discharge from the index admission. For instance, discharge medications were unavailable as were vital signs, radiology and other detailed clinical test results. The diagnosis of acute respiratory failure is also somewhat ambiguous since the cause for respiratory failure could not be definitely ascertained. Acute respiratory failure could result from an inadequately treated COPD exacerbation, pneumonia, pulmonary embolism or another unspecified lung condition. Still, it is likely that in many cases it represents a process different from COPD that could have resulted from a missed diagnosis or inadequate treatment. Additionally, the PHC4 database only includes rehospitalizations for where the original hospitalization occurred. However, the sheer number of hospitalizations analyzed in this dataset outweigh these limitations.

\section{Conclusions}

We show that COPD patients rehospitalized for comorbid conditions such as myocardial infarction, pneumonia or acute renal failure are significantly more likely to die within 30 days than patients readmitted for recurrent COPD exacerbation. More attention should be focused on preemptive risk reduction for these comorbidities in COPD patients to decrease morbidity, mortality and need for rehospitalization.

\section{Declaration of Interest}

The authors have no conflicts of interest to declare including financial and consulting relationships. 


\section{References}

1. Mannino DM. COPD epidemiology, prevalence, morbidity and mortality and disease heterogeneity. Chest. 2002;121(5 Suppl):121S-126S. doi: http://dx.doi.org/10.1378/chest.121.5_suppl.121S

2. Mapel DW, Hurley JS, Frost FJ, Petersen HV, Picchi MA, Coultas DB. Health care utilization in chronic obstructive pulmonary disease. A case-control study in a health maintenance organization. Arch Intern Med. 2000;160(17):2653-2658. doi: http://dx.doi.org/10.1001/archinte.160.17.2653

3. Mannino DM, Buist AS. Global burden of COPD: risk factor, prevalence, and future trends. Lancet. 2007;370(9589):765-773. doi: http://dx.doi.org/10.1016/S0140-6736(07)61380-4

4. Hansell AL, Walk JA, Soriano JB. What do chronic obstructive pulmonary disease patients die from? A multiple cause coding analysis. Eur Respir J. 2003;22(5):809-814.

doi: http://dx.doi.org/10.1183/09031936.03.00031403

5. Mannino DM, Thorn D, Swensen A, Holguin F. Prevalence and outcomes of diabetes hypertension, and cardiovascular disease in COPD. Eur Respir J. 2008;32(4):962-969. doi: http://dx.doi.org/10.1183/09031936.00012408

6. Antonelli Incalzi R, Fuso L, De Rosa M, et al. Co-morbidity contributes to predict mortality of patients with chronic obstructive pulmonary disease. Eur Respir J. 1997;10(12):27942800. doi: http://dx.doi.org/10.1183/09031936.97.10122794

7. Divo M, Cote C, de Torres JP, the BODE Collaborative Group, et al. Comorbidities and risk of mortality in patients with chronic obstructive pulmonary disease. Am J Respir Crit Care Med. 2012;186(2):155-161.

doi: http://dx.doi.org/10.1164/rccm.201201-0034OC

8. Molinos L, Clemente MG, Miranda B, et al, for the ASTURPAR group. Community-acquired pneumonia in patients with and without COPD. J Infect. 2009;58(6):417-424.

doi: http://dx.doi.org/10.1016/j.jinf.2009.03.003

9. Restrepo MI, Mortensen EM, Pugh JA, Anzueto A. COPD is associated with increased mortality in patients with community acquired pneumonia. Eur Respir J. 2006;28(2):346-351.

doi: http://dx.doi.org/10.1183/09031936.06.00131905 